\title{
Kognitive Verhaltenstherapie kann helfen
}

Fragestellung: Hilft kognitive Verhaltenstherapie bei Angst im Rahmen einer demenziellen Erkrankung?

Hintergrund: Angst ist ein häufiges Phänomen bei Demenzen, mit Prävalenzen zwischen 5\% und $21 \%$ für Angststörungen und bis $71 \%$ für Angst als Symptom. Angst kann sich als motorische Unruhe zeigen, aber auch als Agitiertheit, Störung des TagNacht-Rhythmus und/oder Aggression, und, wenn die Demenz fortschreitet, mit zunehmender Pflegebedürftigkeit und Verhaltensstörungen. Im Gegensatz dazu wird selten aktiv nach Angst gefragt, wenn ja werden die Patienten oft mit Psychopharmaka behandelt (wie Antidepressiva), obwohl der Nutzen gering ist. Demgegenüber stehen die Risiken und unerwünschten Arzneiwirkungen.

Kognitive Verhaltenstherapie (KVT) ist eine psychologische Therapiemethode, die Zusammenhänge zwischen Kognition, Gefühlen und Verhalten analysiert und behandelt. KVT ist evidenzbasiert die Methode der Wahl bei Angststörungen älterer Patienten ohne Demenz. KVT wird international als nichtmedikamentöse Methode empfohlen, auch in der Primärversorgung oder in Pflegeheimen Spector A, Charlesworth G, King $M$ et al. Cognitivebehavioural therapy for anxiety in dementia: pilot randomised controlled trial. Br J Psychiatry 2015; 206: $509-16$ bei Depression oder Ängsten. Es gibt Hinweise dafür, dass auch Demenzkranke Fertigkeiten erlernen können. Dies spricht dafür, dass auch hier KVT eingesetzt werden kann, ähnlich wie bei Patien- ten mit Intelligenzminderung. Hinweise für die Machbarkeit von KVT für Ängste und Depression bei der Demenz stammen hauptsächlich aus Fallstudien und zwei kleineren US-amerikanischen randomisierten Studien. In den Studien wurde ein Manual erarbeitet, um die Machbarkeit in einem einfach verblindeten Pilotversuch im Vergleich zur Standardtherapie zu untersuchen. Dabei wurden die Akzeptanz, Adhärenz/Compliance, Rekrutierung, Durchführung und Kosten der Therapie geprüft.

Patienten und Methodik: In der randomisierten kontrollierten Pilotstudie wurde in Phase 1 in einem Gruppenprozess ein Manual entwickelt und in Phase 2 die Machbarkeit randomisiert, einfach verblindet und multizentrisch untersucht. Die 50 ambulanten Teilnehmer hatten eine leichte bis mittelschwere Demenz und einen Score von 11 oder mehr auf der Rating Anxiety in Dementia Scale (RAID; Maximum 54 Punkte). Es musste einen Angehörigen geben und es durften keine schwereren Kommunikationsstörungen vorliegen.

Ergebnisse: Nach 15 Wochen gab es im Vergleich zur Standardbehandlung eine nicht signifikante Änderung von durchschnittlich 3,1 Punkten auf der RAID. Die Änderung der Depressivität war signifikant.

Schlussfolgerungen: Bei der leichten bis mittelschweren Demenz mit Ängstlichkeit ist die Rekrutierung, Akzeptanz und Durchführung einer randomisierten Studie zur kognitiven Verhaltenstherapie möglich.

\section{- Kommentar von Markus Weih, Nürnberg}

\section{Noch ein weiter Weg zur Psychotherapie bei Demenz in der Praxis}

Kausale Fortschritte bei der Therapie der Demenz im Allgemeinen und bei der Demenz vom Alzheimer-Typ im Besonderen lassen weiter auf sich warten. So bedauerlich das ist, desto mehr gute Studien zu nichtmedikamentösen Verfahren und zu den assoziierten Verhaltensstörungen werden publiziert. Dies ist auch dringend nötig, denn gerade Angst, Unruhe, Aggressivität und Depressivität sind oft die Alltagsprobleme der Pflegekräfte und der Angehörigen. Dazu kommt, dass Benzodiazepine und trizyklische Antidepressiva heutzutage bei der Demenz eigentlich obsolet sind und selbst mit SSRI sollte man vorsichtig umgehen. Da die kognitive Verhaltenstherapie (KVT) gut wirksam ist bei Angsterkrankungen, lag die Frage nahe, ob diese Methode trotz Demenz nicht dennoch wirksam sein könnte. Dies muss noch offen bleiben, aber Aimee Spector und ihre Mitarbeiter haben zumindest gezeigt, dass eine Pilotstudie machbar ist. Die vorläufigen Effekte sind allerdings wenig ermutigend: 3,1 Punkte Unterschied auf einer Skala mit 54 Punkten, allerdings sind als "Nebenwirkungen" möglicherweise positive Effekte auf Depressivität zu erwarten. Es muss aber offen bleiben, ob dieser Effekt überhaupt klinisch relevant ist. Dazu kom- men die Kosten der Therapie, die nicht gering sind. Dennoch ist es bei dieser Erkrankung wichtig, jeden noch so kleinen Effekt mangels weiterer Therapieoptionen und wegen der vermutlich größeren unerwünschten Arzneiwirkungen von Psychopharmaka weiter zu verfolgen. Die Demenzkranken sollten nicht anders als andere Gruppen mit Angsterkrankungen behandelt werden. Bis die Psychotherapie bei Demenzkranken in der Praxis aber ankommt, wird es sicher noch lange dauern.

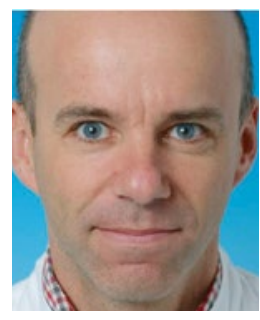

Prof. Dr. med. Markus Weih, Nürnberg

Facharzt für Neurologie, Psychiatrie

und Psychotherapie, Nervenärztliche

Gemeinschaftspraxis, Nürnberg

E-Mail:weih@nervenaerzte-

allersbergerstrasse.de 